\title{
CHEMO-DYNAMICAL MODELS AND THE STAR FORMATION HISTORY OF GALAXIES
}

\author{
M. SAMLAND, G. HENSLER \\ Institut für Astronomie und Astrophysik \\ Universität Kiel, D-24098 Kiel, Germany
}

\section{Introduction}

Sandage (1986) showed what the star formation rate (SFR) of galaxies of different Hubble type might look like. His curves are based on the study of Gallagher et al. (1984), who determined the SFR at three different epochs of galactic evolution. Sandage's figure establishes a connection of SFR and Hubble type but, as was already mentioned by Sandage, it "contains no physics". In order to explore the background of this connection, however, it is necessary either to improve the observations or to model the evolution of galaxies self-consistently. However, the results of modelling the SFR are not reliable, if simplified models are used, which only describe some structural properties of galaxies. It is necessary to apply self-consistent models which take into consideration all relevant interaction processes between the gaseous and stellar components of a galaxy. Such models can be checked by comparison with observations like density and abundance distributions, star-gas content, velocities, velocity dispersions, mass-luminosity relations and age distributions of stars. A detailed model will show, whether the initial conditions, the feedback mechanisms during the evolution or the environment determine the evolution of a galaxy.

\section{Chemo-dynamical Models: A self-consistent way of discribing the evolution of galaxies}

Galaxies consist of complex structures and processes like heating and cooling, dissipation and supernova (SN) are important. In addition, the dynamics of stars and gas influence the evolution. We have developed a 2-d code which describes the dynamics of the stellar and gaseous components, and includes interaction processes like heating and cooling, evaporation and condensation, SNI and SNII, planetary nebulae, dissipation due to cloud-cloud 
collisions, drag, star formation (SF), element synthesis and gravitation, all of which influence the evolution of galaxies. The program named CoDEx (Chemo-Dynamical Evolution of Galaxies) is described in detail in Samland (1994), Samland et al. (1995) and Samland \& Hensler (1995).

As a first application for the chemo-dynamical approach we modelled the evolution of a galaxy, the mass (M) and angular momentum (L) of which is comparable to the Milky Way. If we take into account the observational error margins, our model explains the abundance distributions of nitrogen, oxygen and iron for the stars as well as for the interstellar medium in the different parts of the galaxy. Furthermore, the velocity dispersions of bulge, disk and halo stars can be reproduced. Since the spatial resolution in the model amounts to only 200pc, it is not possible to distinguish between a thick and a thin disk component. Average properties of the disk stars, like the anisotropy of the velocity dispersion, are, however, in agreement with the observations. Detailed results of that model are published in Samland (1994), Samland et al. (1995) and Samland \& Hensler (1995).

\section{The Star Formation History of Galaxies}

Our calculations set in at the time when a gaseous protogalaxy starts cooling and clouds of $10^{5}$ to $10^{7} \mathrm{M}_{\odot}$ are forming. Due to dissipation of kinetic energy the protogalaxy collapses. As feedback mechanisms like SN heating dominate, the collapse time is about a factor 10 longer than the dynamical timescale. The multi-phase character of the ISM also prolongs the collapse time, because hot gas, heated by $\mathrm{SNe}$ is driven out of the star forming regions into the halo.

In our models the halo forms early during a period of $2-3 \cdot 10^{9}$ years, while bulges are on average $2 \cdot 10^{9}$ years younger with a formation time of about $5 \cdot 10^{9}$ years. The disk is the youngest component of a rotating galaxy. While the inner disk forms at the same time as the bulge, the outer disk formation extends over more than $10^{10}$ years. The details of the formation process, in particular the evolution of densities, velocity dispersions, abundances and SFRs are described in Samland (1994) and Samland et al. (1995). It is likely that total mass (Tully et al. 1982), angular momentum (Sandage et al. 1970) and the initial density distribution (Gott \& Thuan 1976) determine the SF history of a galaxy. Because of the complex character of galaxy formation it is, however, not possible to estimate a priori the influence of these quantities on the SFR. Only chemo-dynamical models enable ones to simulate the evolution of different types of galaxies and thus to investigate what determines the global SFR. A number of different models with masses of $0.2-5 \cdot 10^{11} \mathrm{M}_{\odot}$ and different $\mathrm{L}$ have been calculated. For rotating galaxies, independent of their specific angular momentum, we 
derive the relation

$$
\frac{\mathrm{SFR}_{\max }}{\mathrm{M}_{\odot} \mathrm{yr}^{-1}}=16.6 \pm 5.7 \cdot\left(\frac{\mathrm{M}}{10^{11} \mathrm{M}_{\odot}}\right)^{1.5}
$$

For the non-rotating galaxies the dependence remains the same, except that the coefficient 16.6 has to be doubled. Fig. 1 shows the total SFR of four models differing by the total $\mathrm{M}$, total $\mathrm{L}$ and specific angular momentum $\mathrm{L} / \mathrm{M}$ given in the table.

The SF histories of non-rotating galaxies differ from other galaxies because they consume most of their gas during a collapse phase of $2-4 \cdot 10^{9}$ years, so that their later gas content is provided by the stellar mass return. If galaxies are rotating, the collapse is less violent, leading to a lower peak $\mathrm{SF}\left(\mathrm{SFR}_{\max }\right)$ and an increasing gas consumption time. Rotation, however, changes the consumption time and the $\mathrm{SFR}_{\max }$ only by a factor 2 , which is a minor effect compared to the mass dependency of SFR max $_{\text {. We found }}$ no correlation between the Hubble type of a disk galaxy and the total $\mathrm{L}$ or the specific angular momentum. This is confirmed by Miyamoto et al. (1980), who determined the $\mathrm{L}$ and $\mathrm{M}$ of disk and irregular galaxies from observational data. In general the late-type galaxies tend to have lower $M$ than early-type galaxies (Roberts \& Haynes 1994). The question has to be addressed which additional parameters determine the galaxy type, because galaxies with equal $M$ but of different type are observed. As a further example, in Fig.1 we also plotted the SFR of a galaxy (straight line) with $2.2 \cdot 10^{11} \mathrm{M}_{\odot}$ and low specific angular momentum. Contrary to the other galaxies, this model retains a nearly constant SFR for a long time. Evidently, the initial density of this galaxy had been lower and in addition the density distribution was flat.

At the moment, the number of simulations is still too small to conclude how the initial density distribution affects the evolution of galaxies, but we are sure that the precursors of early-type galaxies must have had higher densities in the centre than the ones of the late types. This is consistent with the model of Sofue and Habe (1992) and the observations by Dressler (1979) according to which the environment influences the formation of these different types of protogalaxies and therefore also the galaxies. $M$ of a galaxy also determines the SF history. In Fig.2 we show, how the SFR and the $M$ of two different galaxies evolve in comparison with observational data. The plot clearly reveals the different phases of the galactic evolution. At the beginning, M and SFRs of both galaxies increase due to the collapse. The collapse phase ends when the kinetic energy input of the SNII is of the order of the dissipation rate and when the inflow of cloudy medium is balanced by the outflow of hot gas. Only $10 \%$ of this hot gas is ejected by the SNII, while the rest is contributed by evaporating clouds. The further 


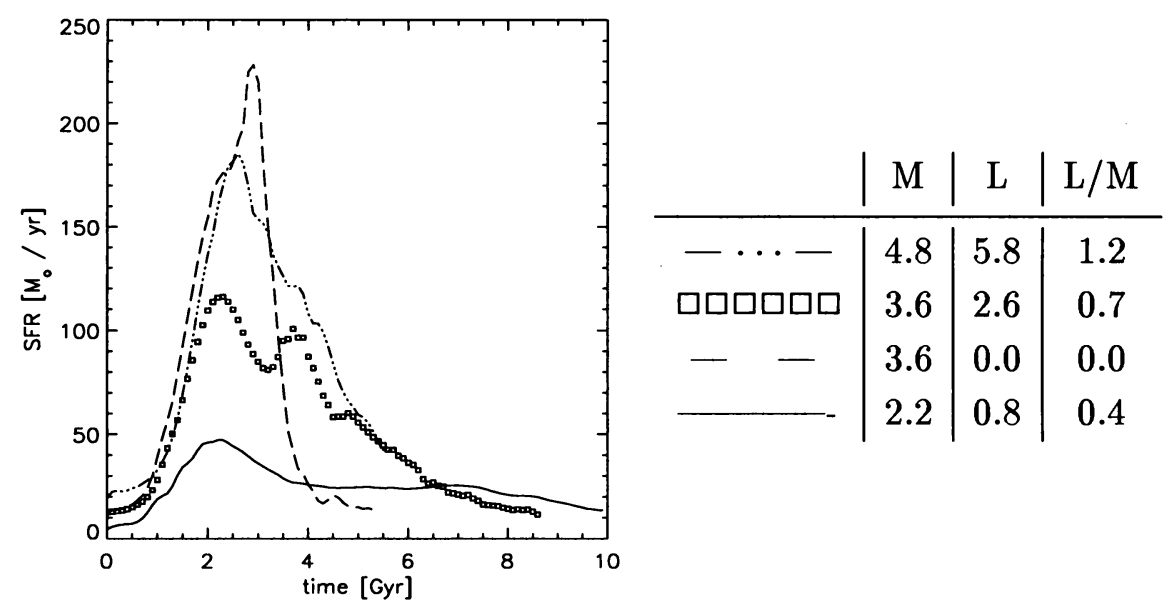

Figure 1. Fig.1: Evolution of the SFR for 4 different galaxies. Mass $\left[10^{11} \mathrm{M}_{\odot}\right]$, angular momentum $\left[10^{11} \mathrm{M}_{\odot} \mathrm{pc}^{2} \mathrm{yr}^{-1}\right]$ and specific angular momentum $\left[\mathrm{pc}^{2} \mathrm{yr}^{-1}\right.$ ] of the models are shown by the table.

evolution depends on the total M. The more massive a galaxy is, the better it can keep the hot gas bound. Therefore the SFR drops with constant M because of gas consumption. The less violent the collapse of a galaxy is, the longer the galaxy needs to convert the clouds into stars. Low-mass galaxies $\left(\mathrm{M}<10^{11} \mathrm{M}_{\odot}\right)$, on the other hand, expel some amount of hot gas by a SN-driven galactic wind. In Fig.2 it can be recognized that $M$ of such a low-mass galaxy decreases during the evolution. It is remarkable that after attaining the maximum the SFR of the low-mass galaxy decreases by one order of magnitude within $5 \cdot 10^{9}$ years, while the more massive galaxy requires $12 \cdot 10^{9}$ years, although the gas fraction of the low-mass galaxy is higher. In order to compare the calculated evolution tracks with the observations, one has to take into account that the errors of the determined SFR and $M$ for individual galaxies are still high and that the data (Hunter \& Gallagher, 1986) are biased towards high SFRs (Some of their objects are classified as starburst galaxies.). Keeping this in mind, we can conclude that the results of the chemo-dynamical models are in good agreement with the observations.

\section{Conclusion}

One of the most important results of the chemo-dynamical approach is that most galaxies evolve as self-regulated systems. We find that the evolution of a galaxy depends strongly on the initial $M$ and the density distribution of the protogalaxy. Compared to the $\mathrm{M}, \mathrm{L}$ plays a minor rôle for the evolution. The simulations predict that early-type galaxies convert most of their 


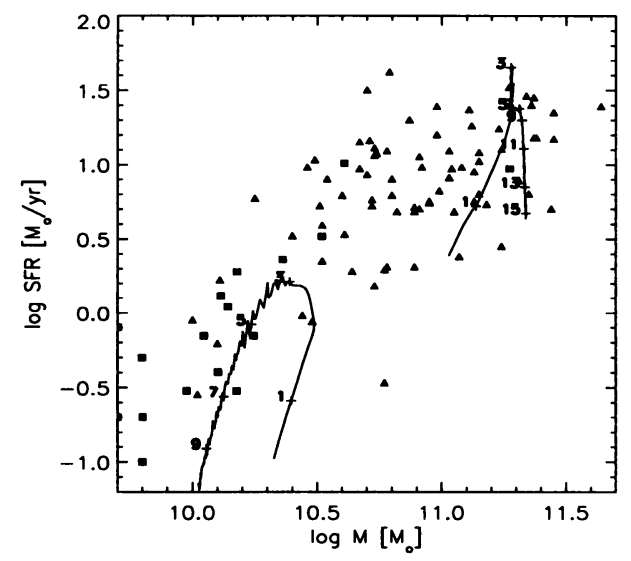

Figure 2. Fig.2: $\mathrm{M}$ and SFR of two models (lines) in comparison with observational data (squares and triangles). The numbers in the plot indicate the age of the galaxies in units of $10^{9}$ years. The squares are data from Hunter \& Gallagher (1986) and the triangles are galaxies from the IRAS bright galaxies catalogue (Condon et al. 1990). Their SFRs are calculated using the formula of Thronson \& Telesco (1986) and their total M are taken from Tully (1988).

gas during an initial collapse phase, which is of the order of $3 \cdot 10^{9}$ years long, and that the late type galaxies convert more gas after the collapse phase. The initial density distribution proposed by Gott \& Thuan (1976) determines the evolution. The precursors of early-type galaxies have higher densities in the centre than the ones of the late types. The evolution of lowmass galaxies is different because they lose mass by galactic winds, what affects the SF history. The chemo-dynamical models show that the SFR of galaxies cannot be described by simple formulae, but in general the results confirm Sandage's ideas concerning the SF history of galaxies.

\section{References}

Condon, J.J., Helou, G., Sanders, D.B., Soifer, B.T., 1990, ApJS 73, 359

Dressler, A., 1979, ApJ 231,659

Gallagher, J.S., Hunter, D.A., Tutukov, A.V., 1984, ApJ 284, 544

Gott, J.R., Thuan, T.X., 1976, ApJ 204, 649

Hunter, D.A., Gallagher, J.S., 1986, PASP 98, 5

Miyamoto, M., Satoh, C., Ohashi, M., 1980, Astrophys. Space Sci. 67, 147

Roberts, M.S., Haynes, M.P., 1994, ARAA 32,115

Samland, M., 1994, Dissertation, University of Kiel, Germany

Samland, M., Hensler, G., 1995, IAU-Symp. 169, in press

Samland, M., Hensler, G., Theis, C., 1995, ApJ submitted

Sandage, A., Freeman, K., Stokes, N., 1970, ApJ 160, 831

Sandage, A., 1986, A\&A 161, 89

Sofue, Y., Habe, A., 1992, PASJ 44,325

Thronson, H.A.J., Telesco, C.M., 1986, ApJ 311,98

Tully, R.B., Mould, J.R., Aaronson, M., 1982, ApJ 257, 527

Tully, R.B., 1988, Nearby Galaxies Catalog, Cambridge University Press 


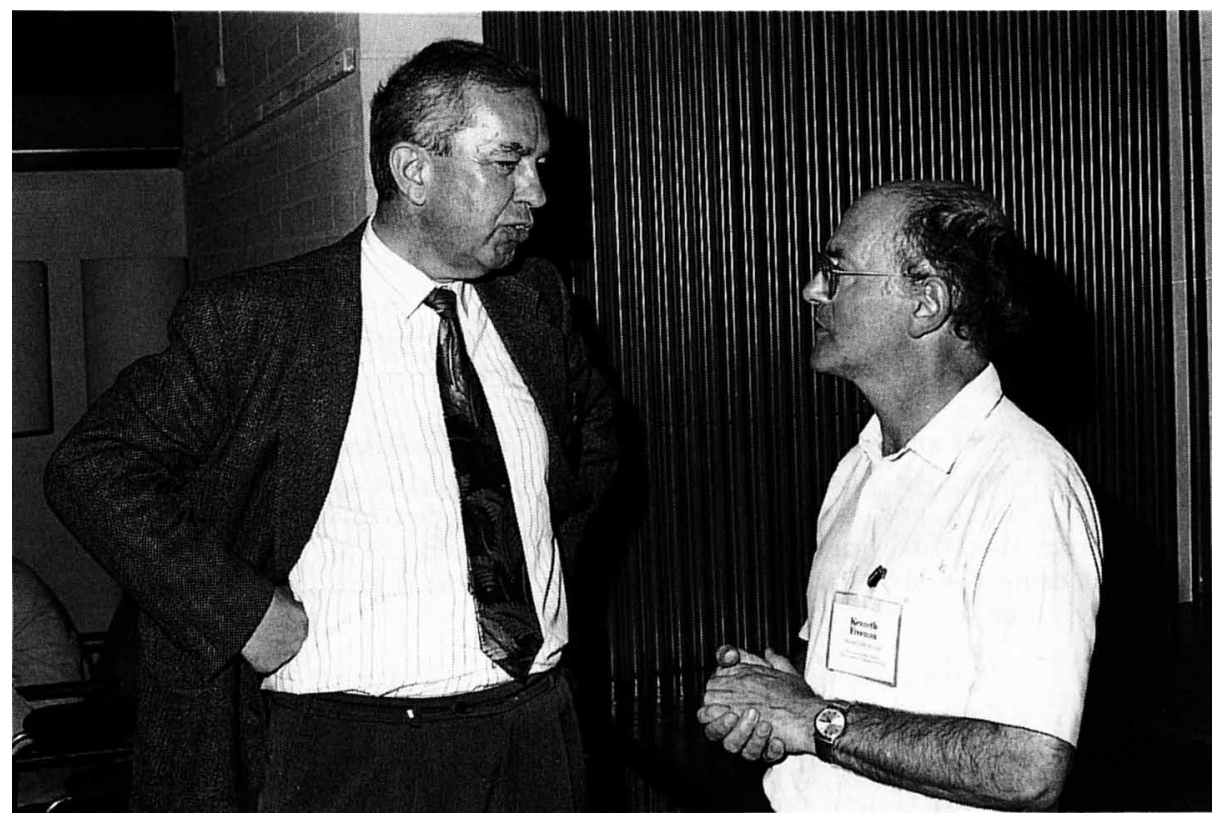

Roland Wielen and Ken Freeman 